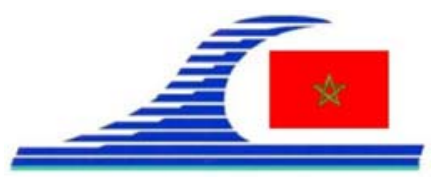

Conférence Méditerranéenne Côtière et Maritime EDITION 2, TANGER, MAROC (2011)

Coastal and Maritime Mediterranean Conference

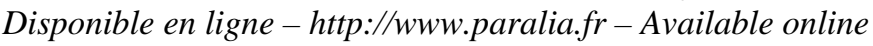

\title{
Contrôle et évolution des milieux sédimentaires de la lagune de Nador (littoral méditerranéen oriental, Maroc)
}

\section{Naima HAMOUMI ${ }^{1}$, Ahmed HOURIMECHE ${ }^{2}$, Moustapha CHAFIK ${ }^{1}$, Mohammed El Amine HAZIM ${ }^{1}$, Loubna TERHZAZ ${ }^{1}$, Rabii KHARBAOUI ${ }^{1}$, Abdelkarim LOUAYA ${ }^{1}$}

1. Laboratoire Océanologie et Géodynamique des Bassins sédimentaires, Faculté des Sciences, Université Mohammed V - Agdal, Rabat, Maroc. naimahamoumi@yahoo.fr

2. Département de Géologie, Faculté des Sciences, Université Hassan II Ben Mssik, Casablanca, Maroc.

\section{Résumé :}

L'étude sédimentologique (analyse stratonomique, analyse granulométrique, calcimétrie et étude minéralogique aux $\mathrm{RX}$ de la roche totale et de la phase argileuse) des dépôts de la lagune de Nador (littoral méditerranéen oriental, Maroc) a porté sur des carottes et des échantillons de surface de la couverture sédimentaire meuble, prélevés au cours de 3 campagnes d'échantillonnage (hiver 2003, été 2004 et hiver 2005), Elle a permis d'identifier les milieux de sédimentation et de suivre leur évolution spatiale pour la période actuelle (hiver 2003, été 2004 et hiver 2005) et pour la première fois pour une période ancienne estimée à 1000-1200 ans BP. Elle a également permis de préciser les sources d'apport, les processus de transport et les facteurs (climat, contexte géologique de l'arrière pays, morphologie et taille de la lagune, position de la passe et activités anthropiques), qui contrôlent les faciès ainsi que la répartition et l'évolution des milieux de sédimentation.

\section{Mots-clés :}

Ecosystème côtier - Sédimentologie - Milieux de sédimentation - Lagune de NadorMaroc - Littoral méditerranéen

\section{Introduction}

La lagune de Nador située dans le littoral méditerranéen oriental (Fig. 1), a une superficie de $115 \mathrm{~km}^{2}$ et une profondeur qui atteint $7 \mathrm{~m}$ dans la partie centrale. Du fait de son intérêt scientifique et socio-économique, elle a fait l'objet de nombreux travaux dont des études sédimentologiques (TESSON \& GENSOUS, 1978 ; IRZI, 1987 ; MAHJOUBI, 2001 ; INANI, 1995 ; DAFIR, 1996 ; LEFEBVRE et al., 1996 ; EL ALAMI et al., 1998 ; MAHJOUBI et al., 2003). Cependant, à l'instar des autres environnements littoraux, la lagune est un milieu fragile dont l'évolution naturelle ou provoquée peut se produire rapidement, et ce d'autant plus qu'elle a été soumise à une pression anthropique importante et souvent anarchique. Il était donc nécessaire 
La connaissance de la Mer :

un vecteur du développement durable en Méditerranée

d'entreprendre une étude sédimentologique détaillée dans le cadre du projet COLASU afin de disposer de données sédimentologiques récentes et contemporaines de celles des masses d'eau et de la microfaune (dans ce volume). Cette étude a pour objectifs de reconstituer pour une période actuelle (2003-2005) et pour la première fois pour une période ancienne (1000-1200 ans BP), les milieux de sédimentation, les sources d'apport, les processus de transport et de sédimentation et les facteurs qui contrôlent la sédimentation à l'échelle saisonnière et à une échelle de l'ordre du millénaire.

\section{Matériels et méthodes}

Les sédiments étudiés 66 carottes et 192 échantillons de surface (Fig. 1) ont été prélevés au cours de 3 campagnes (Hiver 2003, Eté 2004 et Hiver 2005). Le carottage a été effectué par un plongeur à l'aide des tubes en PVC et la position des stations de prélèvement a été déterminée par GPS.

L'étude sédimentologique a porté sur l'analyse stratonomique, l'analyse granulométrique, la calcimétrie et l'étude minéralogique aux RX (roche totale et phase argileuse). Il a été ainsi possible d’identifier les faciès sédimentaires sur la base de leur lithofaciès, leur faciès granulométrique ainsi que leur faciès minéralogique et de suivre leur évolution spatio-temporelle.

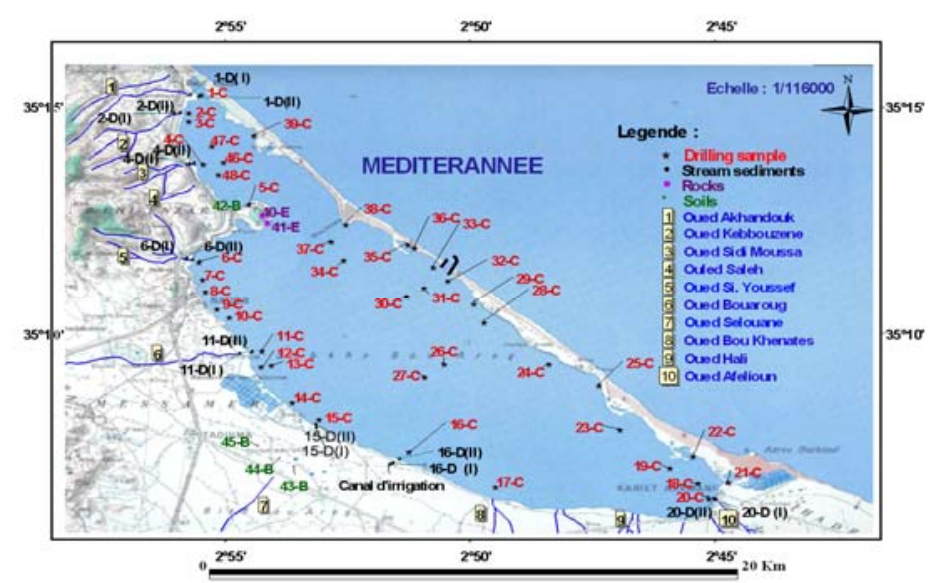

Figure 1. Situation des stations d'échantillonnage des sédiments dans la carte topographique de Nador.

\section{Résultats}

Les faciès sédimentaires identifiés (Fig. 2) ont permis de reconstituer les milieux de sédimentation de la lagune et de suivre leur évolution spatiale pour la période actuelle et pour une période ancienne : 1000 à 1200 ans BP (base des carottes de l'hiver 2003), estimée d'après les datations effectuées par MAHJOUBI (2001). Cinq milieux sédimentaires ont ainsi été reconnus pour la période actuelle :

- le milieu sédimentaire SI sous influence marine très marquée dans la bordure interne de l'île barrière, 
- le milieu sédimentaire SII sous influence marine dans la partie centrale de la lagune,

- le milieu sédimentaire SIII sous influence continentale dans la bordure continentale de la lagune,

- le milieu sédimentaire SIV confiné dans les extrémités NW et SE de la lagune,

- le milieu sédimentaire SV continental dans les embouchures des oueds.

Le contrôle saisonnier ne s'exprime que par une élévation importante de la quantité des lutites dans le milieu SIII de la bordure continentale pendant l'été.

La reconstitution des milieux sédimentaires durant la période 1000 à 1200 ans BP, a permis de mettre en évidence l'existence d'une influence marine à la fois dans l'extrémité NW à Ben Ensar et le Nord de la partie centrale ainsi que des conditions de confinement importants au niveau de l'extrémité SE à Kariat Arekmane. Ces caractéristiques environnementales sont liées à la position de la passe qui était située à cette époque à l'emplacement du double tombolo comme cela est confirmé par les résultats de l'étude écologique (dans ce volume).

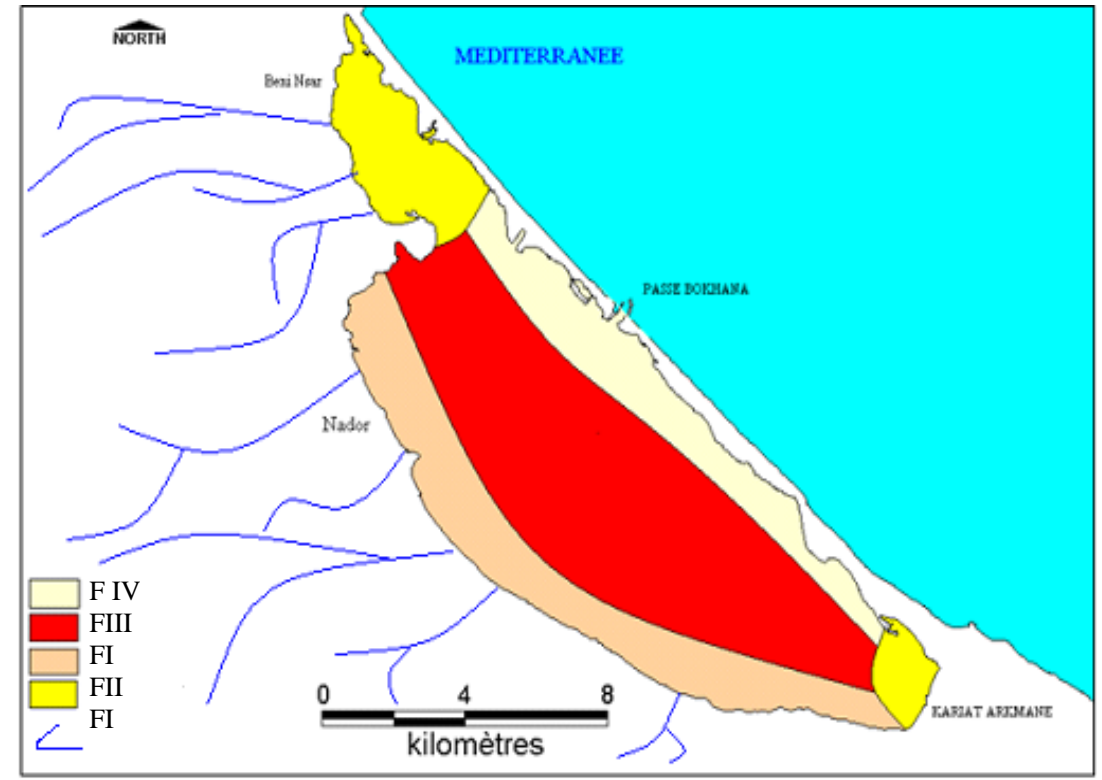

Figure 2. Les faciès sédimentaires de la lagune de Nador durant l'hiver 2003.

La composition des sédiments reflète une alimentation par des processus de transport variés (écoulements à travers la passe, vagues de tempêtes, émissaires à écoulements torrentiels, ruissellements, vents et écoulements gravitaires), à partir de sources intra bassin (faune lagunaire, minerais authigènes) et de sources extra bassin (sédiments marins et produits de l'érosion : des formations géologiques et des sols du bassin versant, de la bordure continentale et de l'île barrière).

Par ailleurs, les faciès ainsi que la répartition et l'évolution des milieux sédimentaires, traduisent un contrôle par le climat, le contexte géologique de l'arrière pays, la 
La connaissance de la Mer :

un vecteur du développement durable en Méditerranée

morphologie et la taille de lagune, la position de la passe et enfin les activités anthropiques qui accentuent le confinement des extrémités NE et SW

\section{Conclusions}

En plus d'un nombre important de données analytiques et de cartes thématiques qui complètent la base de données existantes et peuvent servir d'outil d'aide pour l'aménagement de la région, l'étude sédimentologique des dépôts de la lagune de Nador a permis d'identifier les milieux de sédimentation et de suivre leur évolution spatiale pour la période actuelle et pour la première fois pour une période ancienne estimée à 1000 à 1200 ans BP. Elle a également permis de préciser les sources d'apport, les processus de transport et les facteurs (climat, contexte géologique de l'arrière pays, morphologie et taille de lagune, position de la passe et activités anthropiques), qui contrôlent les faciès ainsi que la répartition et l'évolution des milieux de sédimentation.

\section{Références bibliographiques}

DAFIR J.E. (1996). Organisation et fonctionnement des écosystèmes aquatiques (gestion et préservation). Application de la dynamique du phosphore (flux de fixation /relargage à l'interface eau sédiment, spéciation des différentes fractions mobilisables). Thèse de Doctorat d’Etat ès Sciences, Univ. Hassan II, Casablanca. 320 p.

EL-ALAMI M., MAHJOUBI R., DAMNATI B., KAMEL S., ICOLE M., TAIEB M. (1998). Sédimentologie et géochimie organique des sédiments superficiels de la lagune de Nador (Maroc nord oriental). Journal of African Earth Sciences Vol. 26, Issue 2, pp. 249-259.

INANI I. (1995). Dynamique sédimentaire et état de la pollution dans la lagune de Nador. Thèse de $3^{\text {ème }}$ cycle, Univ. Mohammed V-Agdal, Rabat. 200 p.

IRZI Z. (1987). Etude sédimentologique et micropaléontologique de la lagune de Nador. (Maroc Oriental). Thèse $3^{\text {ème }}$ cycle, Univ. Pierre et Marie Curie, Paris VI, 172 p. LEFEBVRE A., GUELORGET O., PERTHUISOT J.P., DAFIR J. (1996). Evolution biogéologique de la lagune de Nador (Maroc) au cours de la période 1982-1993. Oceanologica Acta, 20, 2, pp. 371-385.

MAHJOUBI R. (2001). Nature et origine du flux de matières particulaires et son enregistrement dans un milieu paralique microtidal : cas de la lagune de Nador (Maroc nord oriental). Thèse de Doctorat d'Etat ès Sciences, Univ Moulay Ismail, Meknès. 273 p.

MAHJOUBI R., KAMEL S., EL MOUMNI B., NOACK Y., PARRON C. (2003). Nature, origine et répartition de la phase argileuse de la lagune de Nador (Maroc Nord oriental. Geologica Belgica 6/1-2, pp.31-42.

TESSON M., GENSOUS B. (1978). Les sédiments superficiels de la lagune de Nador (sebkha de Bou Areg) principales caractéristiques et répartition. Bull Inst Pêches Maroc 24. 